\title{
A Target-Oriented Multiple-Attribute Decision-Making Approach Based on Probabilistic Linguistic Preference Relations
}

\author{
Chun-Hao Li $\mathbb{D}^{1}{ }^{1}$ Hui-Xin Ma $\mathbb{D}^{1},{ }^{1}$ and Yan-Hui Jia ${ }^{2}$ \\ ${ }^{1}$ School of Management, Jilin University, Changchun 130025, China \\ ${ }^{2}$ School of Mechanical and Aerospace Engineering, Jilin University, Changchun 130025, China \\ Correspondence should be addressed to Chun-Hao Li; jyhlichunhao@126.com
}

Received 24 November 2020; Revised 29 December 2020; Accepted 2 January 2021; Published 19 January 2021

Academic Editor: Mingwei Lin

Copyright (C) 2021 Chun-Hao Li et al. This is an open access article distributed under the Creative Commons Attribution License, which permits unrestricted use, distribution, and reproduction in any medium, provided the original work is properly cited.

While the approach to multiple-attribute decision-making (MADM) is widely used in a variety of fields, including models with fuzzy sets and corresponding extensions, it cannot solve target-oriented decision problems with both selective and targeted alternatives. Therefore, this study provides the first description of a target-oriented MADM problem and proposes a novel decision framework. An attribute value function for target orientation is defined by integrating range and frequency values derived under cumulative prospect theory and range-frequency theory. A Choquet integral with discrete fuzzy measures is then used to integrate attribute values and determine comprehensive values for selective alternatives. In this determination of comprehensive values, a parameter estimation model is also established, with its input assumed to be the pairwise comparison judgment matrix with probabilistic linguistic preference relation. This model as well as its transformation aims at determining the parameters of both the attribute value function and fuzzy measures. Finally, the process of target-oriented MADM is summarized, and an illustrative example is provided to demonstrate the applicability of the proposed techniques.

\section{Introduction}

Multiple-attribute decision-making (MADM) involves selecting the best alternative from among a finite set of decision alternatives with multiple attributes [1-3]. It is widely used in a variety of fields including engineering, management, and economics [4-8]. However, due to the uncertainty in the subjective preferences of a decision maker (DM), judgment criteria can be difficult to define [9]. In an attempt to solve this problem, Zadeh proposed the theory of fuzzy sets for application in MADM [10]. Based on it, Torra proposed the hesitant fuzzy set (HFS) as an extension of fuzzy sets, which allowed the DM to provide several possible membership values [11]. Rodríguez et al. further developed hesitant fuzzy linguistic term sets (HFLTS) for a more robust presentation of the linguistic information provided by the DM [12]. HFS and HFLTS both assume that all possible linguistic terms exhibit equal membership degrees [13]. However, in practical applications, various degrees typically exist among possible linguistic terms [14]. Thus, Pang et al. proposed the probabilistic linguistic term set (PLTS), which could represent different membership degrees of all possible evaluation terms (linguistic terms) for a specific alternative [15].

It is often difficult to identify evaluation terms directly when using linguistic variables to describe the DM's judgment [16]. Instead, linguistic preference relation (LPR) provides a better tool for collecting preference information. Jiang and Liao classified LPR into three categories [17]. The first is linguistic term-based LPR, as described by Herrera et al., in which each element is denoted by a linguistic term such as "good" or "very good" [18]. Since this category of LPR is similar to natural language, it is convenient for the DM and only involves a low load of cognition. As such, it is applied in multiple fields. Particularly, Xu et al. proposed a methodology based on LPR for effectively expressing and fusing incomplete linguistic information in large-group emergency decision-making [19]. Chang et al. utilized incomplete LPRs to implement enterprise resource planning [20]. Tan et al. used LPR in the group decision-making of 
supplier selection [21]. The second category is hesitant fuzzy linguistic preference relation (HFLPR), introduced by Zhu and $\mathrm{Xu}$ [22]. It is especially applicable to situations in which the DM is hesitant about more than one linguistic term and is received increased attention in recent years. Specifically, $\mathrm{Li}$ et al. proposed a new hesitant consistency measure, called the interval consistency index, to estimate the consistency range for HFLPR [23]. Liu et al. introduced a technique for calculating each missing element based on the worst and best consistency index in HFLPR [24]. Zhang and $\mathrm{Wu}$ introduced a multiplicative consistency measure for HFLPR and developed a relevant adjustment procedure to modify inconsistent HFLPR values [25]. The last category is probabilistic linguistic preference relation (PLPR), in which individual elements denoted pairwise comparisons of alternatives provided by the DM in the form of PLTS [26]. PLPR is capable of representing different membership degrees of all possible linguistic terms for a specific alternative over another. As such, it is received increasing attention in the literature. Particularly, Nie and Wang proposed several prospect theory-based consistency recovery strategies using multiplicative PLPRs to manage group decision-making [27]. Zhang et al. explored a consensus process for group decision-making with PLPR [28]. Song and Hu used PLPR with incomplete probabilities to manage large-scale group decision problems in complex environments [29]. Gao et al. proposed an emergency decision support methodology based on the multiplicative consistency of PLPR [30]. These studies have made great contribution to the field of MADM and play an important supporting role in solving practical decision-making problems.

Conventional models represent preference information for the DM on each attribute for alternatives. They also provide a comprehensive evaluation of alternatives for scientific decision-making [31, 32]. It is evident that existing methods are established to evaluate alternatives by combining their performance for several attributes and include only one type of alternative. However, practical MADM problems are often target oriented, which exist not only alternatives to be selected (selective alternatives), but also alternatives to be referred (targeted alternatives). Therefore, it is particularly important to make a comprehensive evaluation of selective alternatives based on the orientation of targeted alternatives, by which the real behavioral preference of the DM should be reflected in the process of decisionmaking. The motivation of this paper is to propose a targetoriented MADM approach based on PLPR.

Firstly, target orientation is included in the MADM process. In practical management scenarios, various types of MADM can be target oriented. Alternatives are comprehensively evaluated and ranked according to the DM's preference information to achieve specific targets (goals) [33]. For instance, housing purchase decisions generally refer to transaction information of signed houses, such as area, price, and location. Online shopping decisions generally refer to transaction information of sold products, such as price, mode, and consumers' reviews. Performance levels of each attribute (attribute levels) for targeted alternatives can provide valuable information to be referred in judging attribute values of selective alternatives. Thus, the proposed approach uses a set of targeted alternatives as a reference to make evaluations on selective alternatives.

Secondly, attribute values for alternatives are defined by incorporating range and frequency values [34]. The range value for a specific attribute is a numerical normalization of the subjective value for a specific attribute level, relative to the range of attribute levels. The frequency value is the cumulative probability of a specific attribute level, relative to the distribution of attribute levels [35]. When evaluating alternative attribute values, the derived attribute is often unreasonable if only one type of value is considered while the other is ignored. Taking fairness theory as an example, the degree of satisfaction (value) a clerk attributed to their wage depends not only on the absolute amount (the range value), but also the relative amount compared to other clerks (the frequency value). Thus, even if the absolute wage is high, an individual may feel unsatisfied with their wage if it is comparatively lower than that of their coworkers. Consequently, both range and frequency values should be considered when evaluating attribute values for alternatives [36]. Based on range-frequency theory (RFT) and cumulative prospect theory $(\mathrm{CPT})$, this study constructs a value function of alternatives for each attribute involving both range and frequency values.

Thirdly, PLPR is introduced to estimate parameters for target-oriented MADM. The attribute value function constructed in this study integrates multiple factors such as reference point, range value, and frequency value; it is thus capable of representing the DM's preference information more precisely. However, the attribute value function includes three different parameters (Section 3.2). Accurately estimating the values of these parameters is a key issue in the process of target-oriented MADM, since the orientation of targeted alternatives is represented by the attribute value function. As PLPR can adequately describe the different membership degrees of all possible linguistic terms for a specific alternative, this study applies PLPR to the extraction of the DM's preference information for each attribute. A parameter estimation model is then proposed to derive parameter values using mathematical programming.

The rest of this paper is organized as follows: Section 2 reviews concepts related to PLPR, RFT, and CPT and Choquet integrals. Section 3 proposes a target-oriented MADM approach based on PLPR. Section 4 provides an academic performance evaluation problem and uses the proposed approach to develop a solution. Conclusions are then presented in Section 5.

\section{Preliminary Information}

A target-oriented MADM approach is proposed based on PLPR, RFT, CPT, and Choquet integrals. This section reviews the relevant concepts for each.

2.1. PLPR. Suppose a linguistic term set with an odd granularity is denoted $S=\left\{s_{0}, s_{1}, \ldots, s_{2 \tau}\right\}$. For two linguistic terms, $s_{\alpha}, s_{\beta} \in S$ and $\rho \in[0,1]$, the operational laws $s_{\alpha} \oplus s_{\beta}=$ 
$s_{\alpha+\beta}$ and $\rho s_{\alpha}=s_{\rho \alpha}$ are typically valid [37]. Here, $\oplus$ denotes an aggregation operation between two linguistic terms [38]. Linguistic preference relation (LPR) and its multiplicative consistency are then defined as follows [39].

Definition 1. The matrix $L=\left(l_{i j}\right)_{N \times N}$ represents a LPR if the following conditions hold:

$$
l_{i j} \oplus l_{j i}=s_{2 \tau}, l_{i i}=s_{\tau}, \quad i, j \in N,
$$

where $S=\left\{s_{0}, s_{1}, \ldots, s_{2 \tau}\right\}$ is a given linguistic scale set and $l_{i j}$ denotes the preference degree for an alternative $a_{i}$ over $a_{j}$ [39]. The term $a_{i}$ is preferred to $a_{j}$ if $l_{i j}>s_{\tau}$ and indifferent if $l_{i j}=s_{\tau}$. The LPR condition $L=\left(l_{i j}\right)_{N \times N}$ is multiplicatively consistent if it satisfies the following:

$$
\begin{gathered}
Z\left(l_{i j}\right) \cdot Z\left(l_{j k}\right) \cdot Z\left(l_{k i}\right)=Z\left(l_{i k}\right) \cdot Z\left(l_{k j}\right) \cdot Z\left(l_{j i}\right), \\
i, j, k=1, \ldots, N
\end{gathered}
$$

where $Z(\cdot)$ denotes a subscript function with an inverse defined as $Z^{-1}(\cdot)$.

When the DM simultaneously expresses several linguistic terms in practical applications, he/she may be unable to provide complete linguistic distribution information. In this case, only partial probability information is provided. To better describe such a situation, probabilistic linguistic term set (PLTS) was defined by Pang et al. as follows [15].

Definition 2. Suppose $S=\left\{s_{0}, s_{1}, \ldots, s_{2 \tau}\right\}$ is a linguistic term set. PLTS is then defined as

$$
L(p)=\left\{L_{k}\left(p_{k}\right) \mid L_{k} \in S, p_{k} \geq 0, k=1,2, \ldots, \# L(p), \sum_{k=1}^{\# L(p)} p_{k} \leq 1\right\}
$$

where $L_{k}\left(p_{k}\right)$ represents the linguistic term $L_{k}$ associated with the probability $p_{k}[15]$. The term $\# L(p)$ is the number of different linguistic terms in $L(p)$.

Definition 3. Suppose $S=\left\{s_{0}, s_{1}, \ldots, s_{2 \tau}\right\}$ is a linguistic term set. The expected value for the PLTS term defined in equation (3) can be calculated as [15]

$$
E(L(p))=\sum_{k=1}^{\# L(p)} Z\left(L_{k}\right) \cdot p_{k}
$$

where the definitions of $Z(\cdot)$ and $\# L(p)$ are the same as before.

The DM can provide a PLTS to represent uncertain preference information [40]. For instance, a set $S=\left\{s_{0}, s_{1}, s_{2}\right\}=\{$ somewhat good, good, very good $\}$ is used to indicate the preference for a house. The DM considers the preference level 'good' as the most probable, while the preference level "somewhat good" is more probable than the preference level "very good," which is rare but possible. Thus, the house preference PLTS can be represented by $L(p)=\left\{s_{0}(0.4), s_{1}(0.5), s_{2}(0.1)\right\}=\{$ somewhat good $(0.4)$, good (0.5), very good (0.1)\}. A probabilistic linguistic preference relation (PLPR), proposed by Zhang et al. [26], can then be developed as follows.

Definition 4. The PLPR for the alternative set $A=\left(a_{1}, \ldots, a_{I}\right) \quad$ is represented by a matrix $L=\left(L_{i j}\left(p_{i j}\right)\right)_{N \times N}, i, j=1, \ldots, N$. The term $L_{i j}\left(p_{i j}\right)$ is the preference degree for the alternative $a_{i}$ over $a_{j}$. It satisfies the following conditions [22]:

$$
\left\{\begin{array}{l}
p_{i j}^{(k)}=p_{j i}^{(k)} ; \\
L_{i j}^{(k)}+L_{j i}^{(k)}=s_{2 \tau} ; \\
L_{i i}\left(p_{i i}\right)=s_{0} ; \\
\# L_{i j}\left(p_{i j}\right)=\# L_{j i}\left(p_{j i}\right) ; \\
L_{i j}^{(k)}<L_{i j}^{(k+1)}, L_{j i}^{(k)}>L_{j i}^{(k+1)}, i>j ; \\
p_{i j}^{(k)} \geq 0, \quad \forall i, j ; \\
\# L_{i j}\left(p_{i j}\right) \\
\sum_{k=1}^{(k)} p_{i j}^{(k)}=1,
\end{array}\right.
$$

where $L_{i j}\left(p_{i j}\right)=\left\{L_{i j}^{(k)}\left(p_{i j}^{(k)}\right) \mid k=1, \ldots, \# L_{i j}\left(p_{i j}\right)\right\}$ is the PLTS term based on a given linguistic term set $S=\left\{s_{0}, s_{1}, \ldots, s_{2 \tau}\right\}$ and $L_{i j}^{(k)}$ and $p_{i j}^{(k)}$ are the $k^{\text {th }}$ linguistic term and its corresponding probability in $L_{i j}\left(p_{i j}\right)$, respectively.

2.2. RFT. RFT, proposed by Parducci, asserts the value of a specific attribute level that is determined by two contextual principles: the range of all levels and the cumulative frequency of the level [41]. The range value is a numerical normalization of the subjective value for a specific attribute level, relative to a range of attribute levels. The frequency value is the cumulative probability of a specific attribute level, relative to a distribution of attribute levels.

All decision-making problem attributes are assumed to be benefit attributes, as cost attributes can easily be converted into benefit attributes through normalization. The number of alternatives to be evaluated is represented by $M_{\mathrm{RF}}$, the maximum and minimum attribute levels are denoted $x_{\max }$ and $x_{\min }$, respectively, and the attribute level 
for the alternative is given by $x$. Thus, the attribute value of $x$ in the RFT calculation can be expressed as

$$
V(x)=\delta \cdot v_{\mathrm{RF}}(x)+(1-\delta) \cdot F(x),
$$

where $v_{\mathrm{RF}}(x)$ and $F(x)$ are, respectively, the range and frequency values of $x, \delta$ is the weight corresponding to $v_{\mathrm{RF}}(x)$ and $F(x)$, and $0 \leq \delta \leq 1$. The range value $v_{\mathrm{RF}}(x)$ and the frequency value $F(x)$ are defined below.

Definition 5. Assuming $S(x), S\left(x_{\max }\right)$, and $S\left(x_{\min }\right)$, respectively, represent subjective values for $x, x_{\max }$, and $x_{\min }$, the range value for $x$ relative to the range $\left[x_{\min }, x_{\max }\right]$ is given by [41]

$$
v_{\mathrm{RF}}(x)=\frac{\left[S(x)-S\left(x_{\min }\right)\right]}{\left[S\left(x_{\max }\right)-S\left(x_{\min }\right)\right]} .
$$

Definition 6. The number of alternatives to be evaluated is $M_{\mathrm{RF}}$, and Rank $(x)$ stands for the ranking position of $x$ in an increasing order of all attribute levels. The frequency value of $x$ relative to the frequency of alternatives in the range can then be expressed as [41]

$$
F(x)=\frac{[\operatorname{Rank}(x)-1]}{\left(M_{\mathrm{RF}}-1\right)} .
$$

2.3. CPT and Choquet Integrals. CPT, proposed by Tversky and Kahneman, is the most prominent alternative to expected utility theory for decision-making under risk [42, 43]. It divides the decision-making process into editing and evaluation phases. In the editing phase, the DM collects and processes information based on a decision framework and a reference point. Gains and losses are then defined relative to this reference point. In the evaluation phase, the utility of a prospect is calculated based on a value function and a probability weighting function. The prospect that yields an attribute level $x_{i}$ with a probability $p_{i}(i=1, \ldots, I)$ is denoted by $\left(x_{1}-x, p_{1} ; \ldots ; x_{I}-x, p_{I}\right)$, where $x$. is the reference point. The reference point is typically regarded as the status quo or aspiration level $[43,44]$.

Definition 7. Suppose $\alpha \in(0,1)$ is the value parameter and $\lambda$ is the loss aversion parameter $(\lambda>1)$. The value of $x_{i}$ in CPT can then be calculated from [42]

$$
v_{C P T}\left(x_{i}\right)= \begin{cases}-\lambda \cdot\left[-\left(x_{i}-x_{.}\right)\right]^{\alpha}, & x_{i}<x_{.} \\ \left(x_{i}-x_{.}\right)^{\alpha}, & x_{i} \geq x_{.}\end{cases}
$$

where $x_{i}-x$. represents the gain or loss relative to the reference point and $i=1, \ldots, I$.

Fuzzy measures (also called capacities) can be included in CPT for use in constructing a weight function. In addition, Grabisch et al. demonstrated that Choquet integrals were a suitable aggregation operator when used a fuzzy measure to model the importance of attributes [45].
Definition 8. Let $X=\left\{x_{i} \mid i=1, \ldots, I\right\}$ be a finite set and $h(X)$ be the power set of $X$. A set function $\mu: h(X) \longrightarrow[0,1]$ is then a fuzzy measure if it satisfies the following conditions [46]:

(1) $\mu(\varnothing)=0, \mu(X)=1$,

(2) If $A, B \subseteq X$ and $A \subseteq B$, then $\mu(A) \leq \mu(B)$.

In MADM, $\mu(A)$ can be considered the importance of the attribute set $A$ [47]. The monotonicity of $\mu(A)$ suggests that the importance of a subset of $A$ can increase only when a new attribute is introduced in $A$ [48].

Definition 9. Let $v$ be a positive real-valued function of the set $X=\left\{x_{i} \mid i=1, \ldots, I\right\}$ and $\mu$ be a fuzzy measure on $X$. The discrete Choquet integral model for $v$ with respect to $\mu$ is then expressed as follows [46]:

$$
V_{\mu}(v)=\sum_{i=1}^{n} v\left(x_{(i)}\right)\left[\mu\left(X_{(i)}\right)-\mu\left(X_{(i-1)}\right)\right],
$$

where $(\cdot)$ indicates a permutation of $X$ such that $v\left(x_{(1)}\right) \geq v\left(x_{(2)}\right) \geq \cdots v\left(x_{(I)}\right)$ and $X_{(i)}=\left\{x_{(1)}, \ldots, x_{(i)}\right\}$, with $X_{(0)}=\varnothing$. It is evident from Definition 8 that $\mu(\varnothing)=0$ and $\mu(X)=1$.

The Choquet integral is a subadditive or superadditive integral developed by Choquet [45]. It can be used to represent the correlation of input arguments being aggregated. In recent years, Choquet integrals are used to determine comprehensive values for alternatives by integrating attribute values. Since Choquet integrals can robustly model interactions among attributes, the resulting comprehensive values can more effectively represent the DM's real decision behavior given several alternatives. Corresponding alternatives improve as alternative comprehensive values increase.

\section{The Proposed Method}

3.1. Problem Description. The target-oriented MADM problem can be described as selecting and assessing alternatives using a series of attributes by referring to a set of targeted alternatives. Selective alternatives provide a viable approach to solving specific decision problems or to achieving an expected goal. Targeted alternatives also serve as a reference for evaluating selective alternatives and provide a standard for judging various attributes.

Suppose a set of targeted alternatives $A=\left\{a_{i} \mid i=1, \ldots, I\right\}$ and a set of selective alternatives $B=$ $\left\{b_{j} j=1, \ldots, J\right\}$ are each evaluated using a set of attributes $C=\left\{c_{k} \mid k=1, \ldots, K\right\}$. The levels of the targeted alternative $a_{i}$ and the selective alternative $b_{j}$, relative to attribute $c_{k}$, are denoted $x_{i k}$ and $y_{j k}$, respectively $(\forall i, j, k)$. In MADM, these levels are typically represented by a decision matrix [49]. In this study, the decision matrix of targeted alternatives for all attributes is denoted by $X=\left(x_{i k}\right)_{I \times K}$ and that of selective alternatives is denoted by $Y=\left(y_{j k}\right)_{J \times K}$.

In target-oriented MADM, selective alternatives can achieve the DM's minimum requirements. In other words, 
the level of each attribute for selective alternatives should be equal to or greater than the minimum level of the corresponding attribute for the targeted alternatives. Alternatives that cannot meet these requirements should be excluded from the set of selective alternatives. Selective alternatives whose level for a specific attribute are greater than the maximum level of the corresponding attribute for a targeted alternative can be considered as fully satisfying the target. Thus, their value/utility for a corresponding attribute is equal to that of the maximum level of the targeted alternative [33]. In this situation, the attribute level of a selective alternative can be updated using the maximum level of targeted alternatives. After this preoperation step in targetoriented MADM, there exists such a characteristic in the decision matrix for selective alternatives, denoted $y_{j k} \in\left[\min \left(x_{i k} \mid \forall i\right), \max \left(x_{i k} \mid \forall i\right)\right], \forall j, k$.

It is evident from CPT that the DM, influenced by reference points and subjective preferences, may produce different values with respect to the level of a specific attribute of an alternative. Taking attribute level $x_{i k}=80$ as an example, if the DM's reference point is 60 , he/she has a greater sense of gain than having expected. Otherwise, if the reference point is 100 , he/she has a greater sense of loss than expectation. In addition, the subjective value perceived by the DM, relative to different degrees of gain or loss, will also be affected by the value and loss aversion parameters. For instance, the DM will experience different degrees of preference or aversion for the same gain or loss (e.g., 20).

In target-oriented MADM problems, the DM may evaluate the benefits and limitations of an alternative by taking the targeted alternatives as references or standards. Specifically, the DM may evaluate the values of selective alternatives on each attribute by referring to the value perception of targeted alternatives with corresponding attributes. As discussed in Section 1, the attribute value is related not only to the range value, but also to the frequency value. Consequently, MADM problems under target orientation can be described as follows: (1) An attribute value function is defined to include both range and frequency values under target orientation, using a decision matrix of targeted alternatives $\left(Q_{1}\right)$. (2) The developed attribute value function is used to assess the comprehensive values of selective alternatives in a decision matrix, identifying rankings for each $\left(Q_{2}\right)$. Figure 1 illustrates this description of a targetoriented MADM problem.

\subsection{Attribute Value Functions for Target Orientation.} Based on RFT, a specific attribute value is composed of two weighted components. One is the range value for the specific attribute level relative to the range of all attribute levels. The other is the frequency value for the specific attribute level with respect to the distribution of all attribute levels. This preference is inherent in the needs of the DM. Thus, needhierarchy theory (NHT) can provide a conceptual basis for range and frequency values.

NHT asserts that basic human needs can be represented by a low-to-high hierarchical system, which is composed of physiology, security, affiliation, respect, and self- actualization needs. Higher-tier needs to become activated once an individual is satisfied with lower-tier needs, even though satisfaction does not need to reach $100 \%$ for this to occur. Higher-tier needs can thus coexist with other lowertier needs. It can then be inferred that a specific attribute value may not only satisfy the lowest-tier need (physiology), but also the needs in other higher tiers (e.g., security or affiliation) to various extents. The satisfaction of lower-tier needs is mostly embodied as an inexorable concern and pursuit by the DM, while the satisfaction of higher-tier needs is primarily embodied in the pursuit of precedence over competitors.

RFT reclassifies multiple tiers of the DM's needs into absolute and relative needs, in which the range value represents the satisfaction degree of a specific attribute level for a certain number of lower-tier needs, and the frequency value embodies the satisfaction degree represented by the specific attribute level for one or several higher-tier needs after the lower-tier needs are met. In other words, the range value represents the satisfaction degree of the specific attribute level for the DM's absolute needs, and the frequency value mirrors the satisfaction degree of the specific attribute level, assigning relative precedence. These two constitutions of the attribute value, namely, range value and frequency value, exhibit a weighted connection. In addition, from the perspective of multiple-attribute dimensions, the frequency value for an attribute level in each dimension represents the satisfaction degree for the DM's relative precedent needs. Thus, for a set of attribute levels with different dimensions, these frequency values represent the same preference implication as long as the frequency values are equivalent. Under these conditions, the benchmarks for relative precedence comparisons are guaranteed to be identical.

Without loss of generality, the attribute $c_{k}(k=1, \ldots, K)$ can be set as a benefit attribute (cost attributes can easily be converted into benefit attributes through normalization) and the level of attribute $c_{k}$ for targeted alternatives can be denoted $x_{i k}$. There are a total of $I_{k}$ different attribute levels of $c_{k}$ among the total targeted alternatives. They are sorted from low to high as $x_{1}^{k}, \ldots, x_{I_{k}}^{k}$. The term $x^{k}$ is the reference point for attribute $c_{k}$ determined by the DM $\left(x^{k} \leq x_{1}^{k}\right)$. By reference to RFT, lower-tier needs (such as simple physiology or security needs) are considered a direct pursuit or an absolute pursuit of an attribute level by the DM. Higher-tier needs (including security and affiliation needs) are considered a relative pursuit (pursuit of precedence relative to competitors) of an attribute level by the DM. As such, $\left[x^{k}, x_{1}^{k}\right)$ is denoted the subinterval for targeted attribute levels that can only satisfy absolute needs, and $\left[x_{1}^{k}, x_{I_{k}}^{k}\right]$ is considered the subinterval for targeted attribute levels that can satisfy both absolute and relative needs. As discussed in Section 3.1, target-oriented MADM includes characteristics in the decision matrix for selective alternatives, such as $y_{j k} \in\left[\min \left(x_{i k} \mid \forall i\right), \max \left(x_{i k} \mid \forall i\right)\right](\forall j, k)$. Since $x_{1}^{k}=\min$ $\left(x_{i k} \mid \forall i\right)$ and $x_{I_{k}}^{k}=\max \left(x_{i k} \mid \forall i\right)$, this implies $y_{j k} \in\left[x_{1}^{k}, x_{I_{k}}^{k}\right](\forall j, k)$. Therefore, we need only to acquire an attribute value function on the interval $\left[x_{1}^{k}, x_{I_{k}}^{k}\right]$ to satisfy such a requirement for decision-making, as determined by attribute values for selective attributes. 


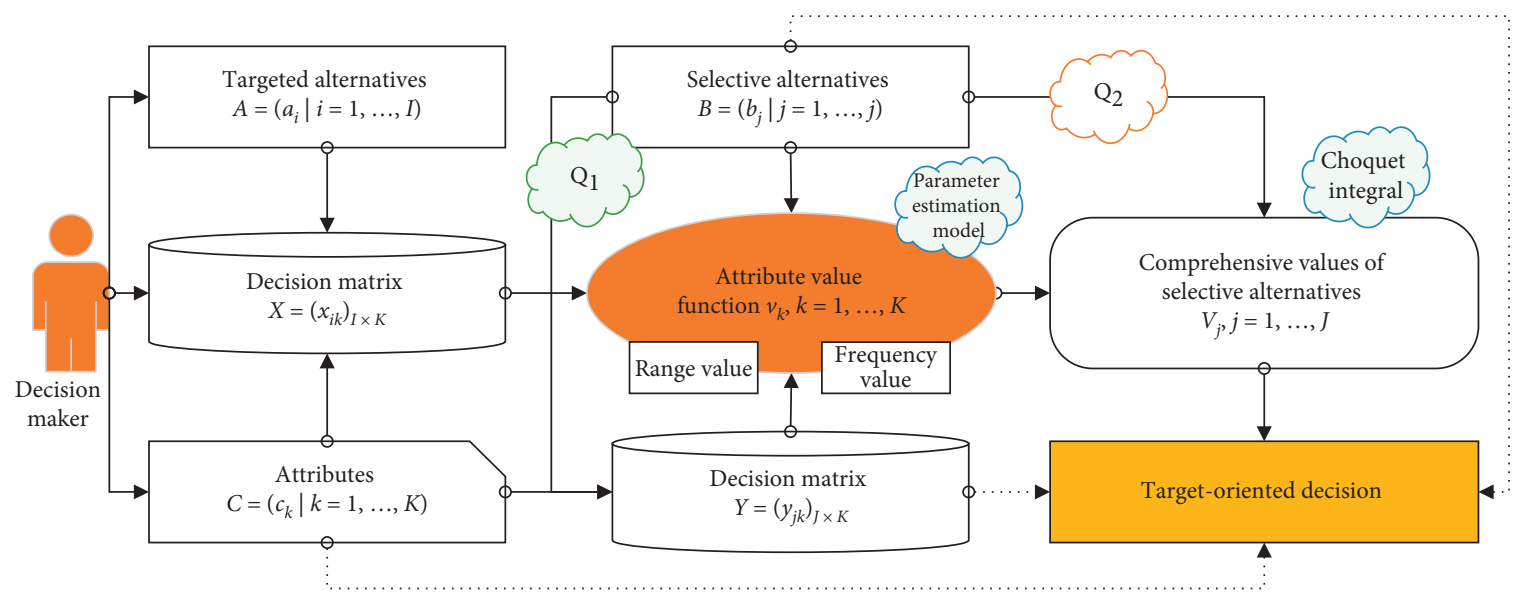

Figure 1: An illustration of the target-oriented MADM problem.

The ratio of targeted alternatives with attribute levels equal to $x_{i_{k}}^{k}\left(i_{k}=1, \ldots, I_{k}\right)$ to total targeted alternatives defines the occurrence probability for $x_{i_{k}}^{k}$. It is denoted $p\left(x_{i_{k}}^{k}\right)$, such that $\sum_{i_{k}=1}^{I_{k}} p\left(x_{i_{k}}^{k}\right)=1$. The term $\pi_{k}=\left[p\left(x_{1}^{k}\right), \ldots, p\left(x_{I_{k}}^{k}\right)\right]$ is defined as the probability distribution of $x_{1}^{k}, \ldots, x_{I_{k}}^{k}$ on $\left[x_{1}^{k}, x_{I_{k}}^{k}\right]$. The cumulative probability of $x_{i_{k}}^{k}$ for targeted alternatives is denoted $F_{k}\left(x_{i k} \mid \pi_{k}\right)$.
It is evident from the definition of $p\left(x_{i_{k}}^{k}\right)$ that $f_{k}\left(x_{i k} \mid \pi_{k}\right)=p\left(x_{1}^{k}\right)+\cdots+p\left(x_{i_{k}}^{k}\right), \quad$ where $i_{k}=1, \ldots, I_{k}$. Based on this definition of $f_{k}\left(x_{i k} \mid \pi_{k}\right)$, the cumulative probability of attribute level $x_{i k}$ for the alternative $a_{i}$, corresponding to the reference distribution $\pi_{k}$ on $\left[x_{1}^{k}, x_{I_{k}}^{k}\right]$, can be calculated as follows:

$$
F_{k}\left(x_{i k} \mid \pi_{k}\right)= \begin{cases}f_{k}\left(x_{i_{k}}^{k} \mid \pi_{k}\right), & x_{i_{k}}^{k} \leq x_{i k}<x_{i_{k}+1}^{k}, i_{k}=1, \ldots, I_{k}-1, \\ 1, & x_{i k}=x_{I_{k}}^{k} .\end{cases}
$$

The attribute value function $v_{k}\left(x_{i k}\right)$ for $x_{i k} \in\left[x_{1}^{k}, x_{I_{k}}^{k}\right]$ on attribute $c_{k}$ can be determined by combining the range and frequency values as follows:

$$
v_{k}\left(x_{i k}\right)=\xi_{k}\left(x_{i k}-x_{.}^{k}\right)^{\alpha_{k}}+\vartheta_{k} F_{k}\left(x_{i k} \mid \pi_{k}\right), \quad x_{1}^{k} \leq x_{i k} \leq x_{I_{k}}^{k} .
$$

This section of the range value is calculated as $\left(x_{i k}-x_{.}^{k}\right)^{\alpha_{k}}$, originating from the definition of value functions in CPT $\left(\alpha_{k} \in(0,1]\right)$. This component of the frequency value is calculated using cumulative probability $F_{k}\left(x_{i k} \mid \pi_{k}\right)$, which is similar to the definition used in RFT. These two regions are represented by the two parameters $\xi_{k}$ and $\vartheta_{k}$, which (along with $\alpha_{k}$ ) can be estimated by the DM (see Section 3.3).

\subsection{Parameter Estimation for Attribute Value Functions.} The attribute value function in equation (12) describes the DM's cognition information for a specific attribute level, described by two aspects of range and frequency values. However, three parameters $\left(\xi_{k}, \vartheta_{k}\right.$, and $\left.\alpha_{k}\right)$ must be estimated. In RFT, a direct scoring method (such as Q-category scale) is typically used to measure attribute values. In this process, $Q$ is frequently set to 5,7 , or 9 and parameter estimation is then conducted using data fitting and other techniques [50]. This approach provides a theoretical reference for parameter estimation in the study.

The attribute value function defines the relationship between any one attribute level (the input) and its corresponding attribute value (the output) [51]. Data fitting-based parameter estimation methods depend not only on the Q-category scale used in estimation, but also on the DM's cognitive ability. Thus, the following two problems must be addressed. First, when the number of attribute values to be estimated is large, it is difficult to distinguish attribute value grades using a Q-category scale, which will affect the accuracy of parameter estimation. Second, this approach fails to consider the limitations of a DM's cognitive ability. If the $\mathrm{DM}$ does not understand the value measurement for a specific attribute, he will often make valuation errors, when required to provide accurate estimation value. To solve these problems, based on PLPR, this study develops a parameter estimation method for attribute value functions.

In the proposed estimation model, a set of representative data points included in the interval $\left[x_{1}^{k}, x_{I_{k}}^{k}\right]$ is introduced and used to estimate parameters. These representative data points are the possible attribute levels between $x_{1}^{k}$ and $x_{I_{k}}^{k}$ whose values can be easily judged by the DM. The modeling of parameter estimation for attribute value functions can be described as follows: these representative data points corresponding to attribute level interval are divided into several 
parts. A pairwise comparison judgment matrix is then introduced to represent the DM's subjective preferences more precisely. Finally, a parameter estimation model is established to determine three parameters: $\xi_{k}, \vartheta_{k}$, and $\alpha_{k}(k=1, \ldots, K)$.

Step 1. Representative data points are selected on the interval $\left[x_{1}^{k}, x_{I_{k}}^{k}\right]$ for attribute $c_{k}$.

A total of $N_{k}$ representative data points are selected, depending on the accuracy of parameter estimation. If the accuracy is required to be high, the value of $N_{k}$ is slightly larger. Otherwise, if the estimation accuracy is lower, the value of $N_{k}$ will be slightly smaller.

Step 2. Divide the representative data points into several groups.

A set of grade scales $S=\left\{s_{q} \mid q=0,1, \ldots, 2 \tau\right\}$ is used to extract the DM's preference for specific attribute levels. For instance, 7 -category scale is denoted $S=\left\{s_{0}\right.$ : very poor, $s_{1}$ : poor, $s_{2}$ : slightly poor, $s_{3}$ : fair, $s_{4}$ : slightly good, $s_{5}$ : good, $s_{6}$ : very good\}. If $N_{k} \leq 2 \tau$, the difference between attribute values for the $N_{k}$ data points could be easily distinguished using the defined grade scales. If $N_{k}>2 \tau$, these differences are more difficult to distinguish using the grade scales. Thus, the $N_{k}$ representative data points should be divided into groups.

Preliminary results suggest grouping the representative data points using the following two steps: (1) Derive the number of groups using $G_{k}=\operatorname{roundup}\left(\left(N_{k}-1\right) / 2 \tau\right)$.

(2) Divide the representative data points into groups and sort from low to high. The first $2 \tau+1$ points form the first group, the points between $2 \tau+1$ and $4 \tau+1$ form the second group, and so on. The remaining points form the $G_{k}$ group are represented by $X_{g_{k}}^{k}=$ $\left(x_{t}^{g_{k}} \mid t=1, \ldots, T_{g_{k}}\right)$ and $g_{k}=1, \ldots, G_{k}$. For instance, assume the points sorted from low to high are given by $x_{1}, \ldots, x_{12}$. If 5 -category scale is used to divide the groups ( $\tau=2$ in this case), the number of groups is $G_{k}=$ roundup $((12-1) / 2 \tau)=3$ and the three groups can be represented as $X_{1}^{k}=\left(x_{1}, \ldots, x_{5}\right), X_{2}^{k}=\left(x_{5}, \ldots, x_{9}\right)$, and $X_{3}^{k}=\left(x_{9}, \ldots, x_{12}\right)$. Specifically, a data cut-off point exists between two adjacent groups (such as $x_{5}$ and $x_{9}$ ), also called the associated attribute level, whose value functions can be used to establish the relationship between attribute values for adjacent groups.

Step 3. Construct a judgment matrix for each group of points by using PLPR.

The DM is provided (in advance) with a probability distribution $\pi_{k}=\left[p\left(x_{1}^{k}\right), \ldots, p\left(x_{I_{k}}^{k}\right)\right]$, representing the range and frequency distributions of targeted alternatives for attribute $c_{k}$. Using this probability distribution $\pi_{k}$ as the decision criterion, the DM is then invited to make pairwise comparisons of values for data points in the existing group $X_{g_{k}}^{k}=\left(x_{t}^{g_{k}} \mid t=1, \ldots, T_{g_{k}}\right)$. The DM's cognitive judgment is reflected by applying PLPR to the set of grade scales $S=\left\{s_{q} \mid q=0,1, \ldots, 2 \tau\right\}$, introduced to express relative preference degrees. These preferences for the data point $x_{t}^{g_{k}}$ over $x_{t^{\prime}}^{g_{k}}$ are given by $L_{t t^{\prime}}^{g_{k}}(p)=\left\{L_{t t^{\prime}, q}^{g_{k}}\left(p_{t t^{\prime}, q}^{g_{k}}\right) \mid L_{t t^{\prime}, q}^{g_{k}} \in S, p_{t t^{\prime}, q}^{g_{k}} \geq 0, q=0, \ldots\right.$, $2 \tau\}$, and the judgment matrix for the DM is constructed as follows:

$$
L_{g_{k}}=\left(L_{t t^{\prime}}^{g_{k}}(p)\right)_{T_{g_{k} \times T_{g_{k}}}}=\left(\begin{array}{cccc}
1 & L_{12}^{g_{k}}(p) & \cdots & (p) \\
L_{21}^{g_{k}}(p) & 1 & \cdots & (p) \\
\vdots & \cdots & \ddots & \vdots \\
L_{T_{g_{k}}}^{g_{k}}(p) & L_{T_{g_{k}}}^{g_{k}}(p) & \cdots & 1
\end{array}\right)
$$

The probability distribution $\pi_{k}$ is used as the decision criterion to provide sufficient information for the DM to make comparison judgment for attribute values, from the integration perspective of range and frequency values. PLPR can then be used to provide relative judgment information and truly reflect the DM's subjective cognitive capability. The term $L_{t t^{\prime}}^{g_{k}}(p)$ denotes the preference degree for the data point $x_{t}^{g_{k}}$ over $x_{t^{\prime}}^{g_{k}}$. If the DM can provide an accurate judgment, $L_{t t^{\prime}}^{g_{k}}(p)$ can be simplified to a scale that is similar to scale values in the AHP judgment matrix [52]. Otherwise, if the DM cannot make an accurate judgment, $L_{t t^{\prime}}^{g_{k}}(p)$ can also be used to describe the DM's preference probability distribution for various scales.

Step 4. Construct a parameter estimation model.

According to Definition 3, the expected value of $\operatorname{PLTS} L_{t t^{\prime}}^{g_{k}}(p)$ can be calculated from $e_{t t^{\prime}}^{g_{k}}=E\left(L_{t t^{\prime}}^{g_{k}}(p)\right)=\sum_{q=0}^{2 \tau} Z\left(L_{t t^{\prime}, q}^{g_{k}}\right) \cdot p_{t t^{\prime}, q^{\prime}}^{g_{k}}$, where $Z(\cdot)$ denotes a subscript function and $\forall t, t, g_{k}$. Equation (2) suggests the PLPR $\left(L_{g_{k}}\right)$ expressed in equation (13) has an expected multiplicative consistency if $e_{t t^{\prime}}^{g_{k}} \cdot e_{t^{\prime} t^{\prime \prime}}^{g_{k}} \cdot e_{t^{\prime \prime} t}^{g_{k}}=e_{t t^{\prime \prime}}^{g_{k}} \cdot e_{t t^{\prime}}^{g_{k}} \cdot e_{t^{\prime} t}^{g_{k}}\left(\forall t, t^{\prime}, t^{\prime \prime} \in\left\{1, \ldots, T_{g_{k}}\right\}\right)$. The following property then emerges: 


$$
e_{t t^{\prime}}^{g_{k}}=\sum_{q=0}^{2 \tau} Z\left(L_{t t^{\prime}, q}^{g_{k}}\right) \cdot p_{t t^{\prime}, q}^{g_{k}}=2 \tau \cdot\left[\frac{w_{t}^{g_{k}}}{\left(w_{t}^{g_{k}}+w_{t^{\prime}}^{g_{k}}\right)}\right], \quad t, t^{\prime}=1, \ldots, T_{g_{k}}, q=0, \ldots, 2 \tau
$$

Since the PLPR $\left(L_{g_{k}}\right)$ ) is not always completely consistent, deviations may exist between the expected value $e_{t t^{\prime}}^{g_{k}}$ and $2 \tau \cdot\left[w_{t}^{g_{k}} /\left(w_{t}^{g_{k}}+w_{t^{\prime}}^{g_{k}}\right)\right]$. We can describe such deviations in $X_{g_{k}}^{k}$ as follows:

$$
\varepsilon_{g_{k}}=\sum_{t=1}^{T_{g_{k}}-1} \sum_{t^{\prime}=2, t^{\prime}>t}^{T_{g_{k}}}\left|\left(e_{t t^{\prime}}^{g_{k}}-2 \tau\right) \cdot w_{t}^{g_{k}}+e_{t t^{\prime}}^{g_{k}} \cdot w_{t^{\prime}}^{g_{k}}\right|
$$

Substituting the definition of $e_{t t^{\prime}}^{g_{k}}$ into this expression yields

$$
\begin{aligned}
\mathcal{E}_{g_{k}}= & \sum_{t=1}^{T_{g_{k}}-1} \sum_{t^{\prime}=2, t^{\prime}>t}^{T_{g_{k}}} \mid w_{t}^{g_{k}} \cdot\left(\sum_{q=0}^{2 \tau} Z\left(L_{t t^{\prime}, q}^{g_{k}}\right) \cdot p_{t t^{\prime}, q}^{g_{k}}-2 \tau\right) \\
& +w_{t^{\prime}}^{g_{k}} \cdot \sum_{q=0}^{2 \tau} Z\left(L_{t t^{\prime}, q}^{g_{k}}\right) \cdot p_{t t^{\prime}, q}^{g_{k}} \mid .
\end{aligned}
$$

Deviations in all points $\left(N_{k}\right)$ can be calculated using the sum in each group (i.e., $\varepsilon=\sum_{g_{k}=1}^{G_{k}} \varepsilon_{g_{k}}$ ). Let $w_{t}^{g_{k}}$ be the relative preference weight for data points $x_{t}^{g_{k}}$ in group $X_{g_{k}}^{k}$ and $u_{t}^{g_{k}}$ be the relative preference weight for the data point $x_{t}^{g_{k}}$ in the whole group (data points in all groups) $X^{k}=X_{1}^{k} \cup \cdots \cup X_{G_{k}}^{k}$. The terms $w_{t}^{g_{k}}$ and $u_{t}^{g_{k}}$ have the same connotation; therefore, they exhibit the relationship with associated attribute levels that $w_{t}^{g_{k}} / w_{T^{g_{k}}}^{g_{k}}=u_{t}^{g_{k}} / u_{T_{g_{k}}}^{g_{k}}\left(t=1, \ldots, T_{g_{k}}, g_{k}=1\right) \quad$ and $w_{t}^{g_{k}} / w_{1}^{g_{k}}=\mathfrak{u}_{t}^{g_{k}} / u_{1}^{g_{k}}\left(t={ }^{g_{k}} 1, \ldots, T_{g_{k}}, g_{k}=2, \ldots, G_{k}\right)$.

Since there are associated attribute levels in adjacent groups, the sum of all relative preference weights for data points within the whole group $X^{k}$ can be expressed as $\sum_{g_{k}=1}^{G_{k}} \sum_{t=1}^{T_{g_{k}}} u_{t}^{g_{k}}-\sum_{g_{k}=1}^{G_{k}-1} u_{T_{g_{k}}}^{g_{k}}$. This term is frequently required to equal 1 , to ensure $a_{T}$ single constraint for the data in all groups (i.e., $\sum_{g_{k}=1}^{G_{k}} \sum_{t=1}^{T_{g_{k}}} u_{t}^{g_{k}}-\sum_{g_{k}=1}^{G_{k}-1} u_{T}^{g_{k}}=1$ ). In addition, since the minimum and maximum of attribute values are often defined as 0 and $1, v_{t}^{g_{k}}=$ $\alpha+\beta \cdot u_{t}^{g_{k}}$ allows the relative preference weights of data points $x_{t}^{g_{k}}$ within the whole group $X^{k}$ to satisfy the constraints for two extreme points. Consequently, a parameter estimation model containing two phases for attribute value functions can be developed by minimizing all deviations using the following description:

$$
\begin{aligned}
& \min \varepsilon_{1}=\sum_{g_{k}=1}^{G_{k}} \sum_{t=1}^{T_{g_{k}}-1} \sum_{t^{\prime}=2, t^{\prime}>t}^{T_{g_{k}}}\left|w_{t}^{g_{k}} \cdot\left(\sum_{q=0}^{2 \tau} Z\left(L_{t t^{\prime}, q}^{g_{k}}\right) \cdot p_{t t^{\prime}, q}^{g_{k}}-2 \tau\right)+w_{t^{\prime}}^{g_{k}} \cdot \sum_{q=0}^{2 \tau} Z\left(L_{t t^{\prime}, q}^{g_{k}}\right) \cdot p_{t t^{\prime}, q}^{g_{k}}\right| \\
& \text { s.t. }\left\{\begin{array}{l}
0 \leq w_{t}^{g_{k}} \leq w_{t+1}^{g_{k}}, \quad t=1, \ldots, T_{g_{k}}-1, g_{k}=1, \ldots, G_{k} \\
u_{T_{g_{k}}}^{g_{k}}=u_{1}^{g_{k}+1}, \quad g_{k}=1, \ldots, G_{k}-1 \\
\frac{w_{t}^{g_{k}}}{w_{g_{g_{k}}}^{g_{k}}}=\frac{u_{t}^{g_{k}}}{u_{T_{g_{k}}}^{g_{k}}}, \quad t=1, \ldots, T_{g_{k}}, g_{k}=1 \\
\frac{w_{t}^{g_{k}}}{w_{1}^{g_{k}}}=\frac{u_{t}^{g_{k}}}{u_{1}^{g_{k}}}, \quad t=1, \ldots, T_{g_{k}}, g_{k}=2, \ldots, G_{k} \\
\sum_{g_{k}=1}^{G_{k}} \sum_{t=1}^{T_{g_{k}}} u_{t}^{g_{k}}-\sum_{g_{k}=1}^{G_{k}-1} u_{T_{g_{k}}}^{g_{k}}=1,
\end{array}\right.
\end{aligned}
$$

$$
\begin{aligned}
& \min \varepsilon_{2}=\sum_{g_{k}=1}^{G_{k}} \sum_{t=1}^{T_{g_{k}}-1}\left|v_{t}^{g_{k}}-\left(\alpha+\beta \cdot u_{t}^{g_{k}}\right)\right| \\
& \text { s.t. }\left\{\begin{array}{l}
v_{t}^{g_{k}}=\xi_{k} \cdot\left(x_{t}^{g_{k}}-x_{.}^{k}\right)^{\alpha_{k}}+\vartheta_{k} \cdot F_{k}\left(x_{t}^{g_{k}} \mid \pi_{k}\right), \quad t=1, \ldots, T_{g_{k}}, g_{k}=1, \ldots, G_{k} \\
v_{t}^{g_{k}}=0, \quad \text { if } t=1, g_{k}=1 \\
v_{t}^{g_{k}}=1, \quad \text { if } t=T_{g_{k}}, g_{k}=G_{k} .
\end{array}\right.
\end{aligned}
$$


In equation (17a), the first constraint ensures the relative preference weights form an increasing function of data points. The second constraint ensures the relative preference weight for each associated attribute level in the adjacent groups is equal. The third and fourth constraints establish relationships between relative preference weights in each individual group. In equation (17b), a relationship between attribute values and relative preference weights within data point sets is established, and these constraints ensure the attribute values satisfy the normalization requirements (a minimum value of 0 and a maximum value of 1 ).

Step 5. Determine parameters.

The parameter estimation model established in equations (17a) and (17b) can be solved as follows:

$$
\begin{aligned}
& \min \varepsilon_{1}=\sum_{g_{k}=1}^{G_{k}} \sum_{t=1}^{T_{g_{k}}-1} \sum_{t^{\prime}=2, t^{\prime}>t}^{T_{g_{k}}}\left(\leftarrow_{t t^{\prime}}^{g_{k}}+\vec{d}_{t t^{\prime}}^{g_{k}}\right)
\end{aligned}
$$

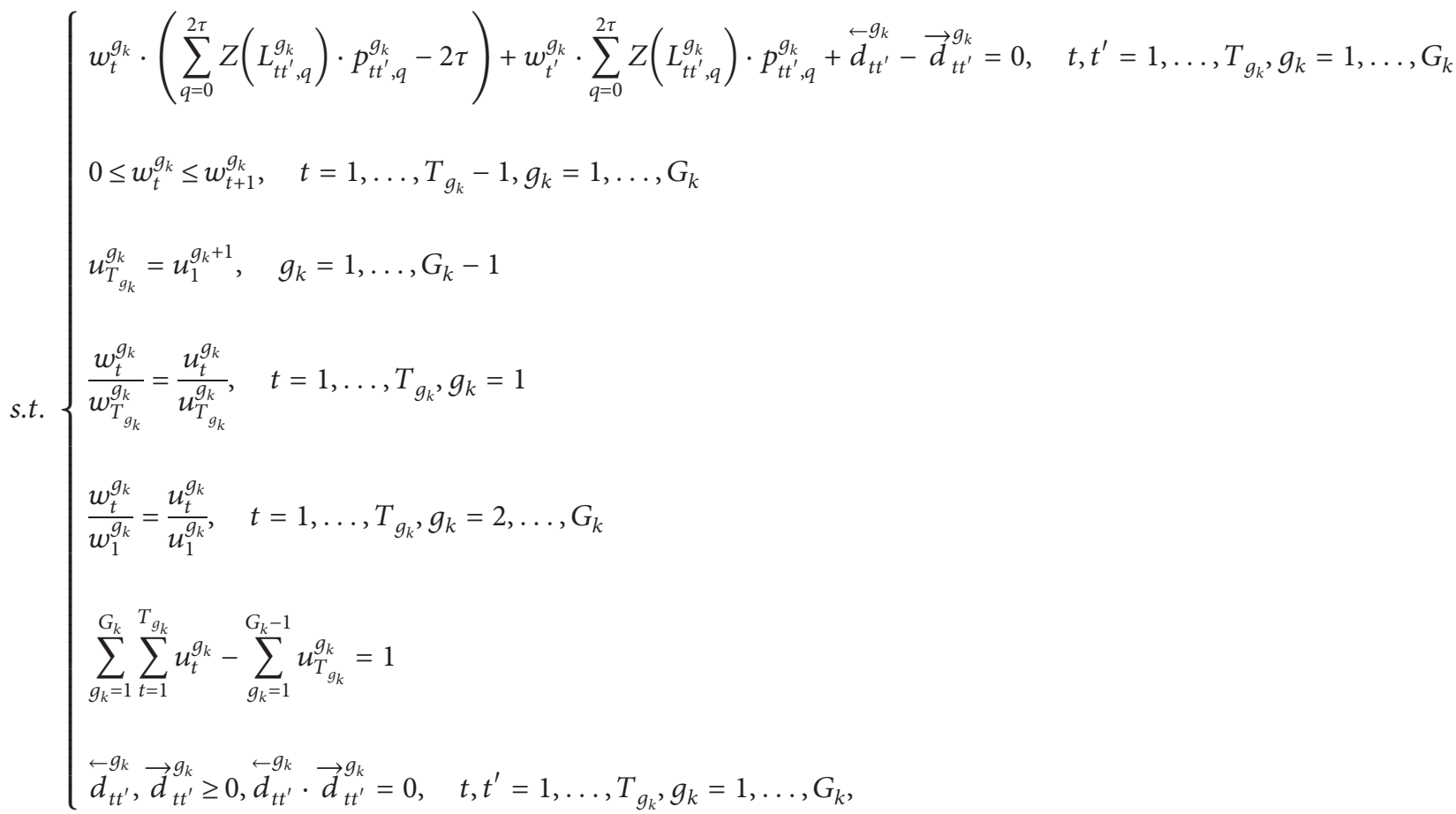

$\min \varepsilon_{2}=\sum_{g_{k}=1}^{G_{k}} \sum_{t=1}^{T_{g_{k}}-1}\left(\stackrel{\leftarrow g_{k}}{t}+\vec{d}_{t}^{g_{k}}\right)$

s.t. $\left\{\begin{array}{l}v_{t}^{g_{k}}=\alpha+\beta \cdot \widehat{u}_{t}^{g_{k}}+\overleftarrow{d}_{t}^{g_{k}}-\vec{d}_{t}^{g_{k}}, \quad t=1, \ldots, T_{g_{k}}, g_{k}=1, \ldots, G_{k} \\ v_{t}^{g_{k}}=\xi_{k} \cdot\left(x_{t}^{g_{k}}-x_{.}^{k}\right)^{\alpha_{k}}+\vartheta_{k} \cdot F_{k}\left(x_{t}^{g_{k}} \mid \pi_{k}\right), t=1, \ldots, T_{g_{k}}, g_{k}=1, \ldots, G_{k} \\ v_{t}^{g_{k}}=0, \quad \text { if } t=1, g_{k}=1 \\ v_{t}^{g_{k}}=1, \quad \text { if } t=T_{g_{k}} g_{k}=G_{k} \\ \stackrel{\leftarrow g_{k}}{d_{t}}, \vec{d}_{t}^{g_{k}} \geq 0, \stackrel{\leftarrow g}{d}_{t} \cdot \vec{d}_{t}^{g_{k}}=0, \quad t, t^{\prime}=1, \ldots, T_{g_{k}}, g_{k}=1, \ldots, G_{k} .\end{array}\right.$

In equation (18a), ${\stackrel{\leftarrow}{d t t^{\prime}}}_{g_{k}}$ and $\vec{d}_{t t^{\prime}}^{g_{k}}$ are the negative and positive deviations with respect to goal $\varepsilon_{1}$, respectively. Similarly, in equation (18b), $\stackrel{\leftarrow}{d}_{t}^{g_{k}}$ and $\vec{d}_{t}^{g_{k}}$ are the negative and positive deviations with respect to goal $\varepsilon_{2}$, respectively. These terms are introduced to establish an equal relationship between ideal and decision solutions. The last constraint in equation (18a) or equation (18b) is used to ensure there is at most one deviation variable greater than zero. The meanings of other constraints are the same as in equations (17a) and (17b).

The model established in equations (18a) and (18b) can be solved using software such as Matlab or Lingo. The 
optimal solutions $\xi_{k}^{*}, \alpha_{k}^{*}$, and $\vartheta_{k}^{*}$ are estimated parameters of the attribute value function for attribute $c_{k}$. The attribute value function represented by equation (12), with the estimated parameters $\xi_{k}^{*}, \alpha_{k}^{*}$, and $\vartheta_{k}^{*}$, can be used to determine the value of selective alternatives for the attribute $c_{k}(k=1, \ldots, K)$.

3.4. Choquet Integral Calculation and Decision-Making. The attribute value function is determined to be $v_{k}\left(x_{i k}\right)=\xi_{k}^{*} \cdot\left(x_{i k}-x^{k}\right)^{\alpha_{k}^{*}}+\vartheta_{k}^{*} \cdot F_{k}\left(x_{i k} \mid \pi_{k}\right)$, using the parameter estimation model in Section 3.3. It is able to represent the DM's value perception of targeted alternatives for each attribute. In target-oriented MADM, the attribute values of selective alternatives $B=\left\{b_{j} \mid j=1, \ldots, J\right\}$ for each attribute can be calculated using the determined value function. Thus, the attribute value of selective alternative $b_{j}$ for attribute $c_{k}$ can be calculated as $v_{k}\left(y_{j k}\right)=$ $\xi_{k}^{*} \cdot\left(y_{j k}-x^{k}\right)^{\alpha_{k}^{*}}+\vartheta_{k}^{*} \cdot F_{k}\left(y_{j k} \mid \pi_{k}\right)$, where $F_{k}\left(y_{j k} \mid \pi_{k}\right)$ is given by

$$
F_{k}\left(y_{j k} \mid \pi_{k}\right)=\left\{\begin{array}{ll}
f_{k}\left(x_{i_{k}}^{k} \mid \pi_{k}\right) & x_{i_{k}}^{k} \leq y_{j k}<x_{i_{k}+1}^{k}, i_{k}=1, \ldots, I_{k}-1 \\
1 & y_{j k}=x_{I_{k}}^{k}
\end{array}, \quad j=1, \ldots, J, k=1, \ldots, K .\right.
$$

Alternative values can be used to represent whole levels for all attributes by integrating the attribute values for each selective alternative.

Choquet integrals provide a useful tool for modeling the interdependence or correlation among various attributes [53]. This study used a discrete Choquet integral to integrate values for all attributes in the MADM problem. From Definition $9, v_{k}\left(y_{j k}\right)$ is a positive value function on the attribute set $C=\left\{c_{k} \mid k=1, \ldots, K\right\}$ and $\mu$ is a fuzzy measure on $C$. The discrete Choquet integral of the values for all attributes with respect to $\mu$ can then be expressed as

$$
V_{j}=\sum_{k=1}^{K} v_{k}\left(y_{j(k)}\right) \cdot\left(\mu\left(C_{j(k)}\right)-\mu\left(C_{j(k-1)}\right)\right),
$$

where (.) indicates a permutation of $\left(y_{1}^{k}, \ldots, y_{J_{k}}^{k}\right)$, such that $v_{k}\left(y_{j(1)}\right) \geq \cdots \geq v_{k}\left(y_{j(K)}\right)$ and $C_{j(k)}=\left\{c_{j(1)}, \ldots, c_{j(k)}\right\}$. In addition, $C_{j(0)}=\varnothing$, where $c_{j(k)}$ is an attribute corresponding to $y_{j(k)}$. The term $V_{j}$ is the comprehensive value of the selective alternative $b_{j}(j=1, \ldots, J)$.

The fuzzy measure $\mu$ is typically identified using semantics [46], learning data [47], or other similar techniques $[53,54]$. When the number of alternatives is large, fuzzy measures often adopt a 2-additive Mobius representation to simplify the calculation [55]. When the number of alternatives is relatively small (a common situation in practice), fuzzy measures can be directly identified by the DM or determined from a series of fuzzy calculations, since the number of measures needing to be calculated is at most $J *(K-1)$, including repeated values. Following the principle of semantics approach, we also employ PLPR to determine fuzzy measures. The set of fuzzy measures to be calculated is denoted $\mu(D)=\left(\mu_{r} \mid r=1, \ldots, R-1\right)$ $=\left(\mu\left(D_{(r)}\right) \mid r=1, \ldots, R-1\right)$, where $D_{(r)} \in\left\{C_{j(k)} \mid j=\right.$ $1, \ldots, J, k=1, \ldots, K\}$, and $r=1, \ldots, R-1$. This set includes only those terms that appeared or are used in the calculations, with unrelated terms excluded. A benchmark for making comparisons is established as $\mu_{R}=\mu\left(D_{(R)}\right)=\mu(C)=\mu\left(\left\{c_{1}, \ldots, c_{K}\right\}\right)$. This set of fuzzy measures can then be represented as $\mu(D)=\left(\mu_{r} \mid r=1, \ldots, R\right)$. From Definition 9, it is evident that $\mu_{R}=\mu\left(\left\{c_{1}, \ldots, c_{K}\right\}\right)=1$.

As in Section 3.3, the PLPR on $S=\left\{s_{q} \mid q=0,1, \ldots, 2 \tau\right\}$ is used to express preference degrees. Suppose preference degrees of fuzzy measure $\mu_{r}$ over $\mu_{r^{\prime}}$ is $L_{r r^{\prime}}^{D}(p)=\left\{L_{r r^{\prime}, q}^{D}\left(p_{r r^{\prime}, q}^{D}\right) \mid L_{r r^{\prime}, q}^{D} \in S, p_{r r^{\prime}, q}^{D} \geq 0, q=0, \ldots, 2 \tau\right\}$.

The DM's judgment matrix can then be constructed as follows:

$$
L_{D}=\left(L_{r r^{\prime}}^{D}(p)\right)_{R \times R}=\left(\begin{array}{cccc}
1 & L_{12}^{D}(p) & \cdots & L_{1 R}^{D}(p) \\
L_{21}^{D}(p) & 1 & \cdots & L_{2 R}^{D}(p) \\
\vdots & \cdots & \ddots & \vdots \\
L_{R 1}^{D}(p) & L_{R 2}^{D}(p) & \cdots & 1
\end{array}\right) .
$$

A fuzzy measure determination model is then developed 


$$
\begin{aligned}
& \min \varepsilon=\sum_{r=1}^{R-1} \sum_{r^{\prime}=2, r^{\prime}>r}^{R}\left(\overleftarrow{d}_{r r^{\prime}}^{D}+\vec{d}_{r r^{\prime}}^{D}\right) \\
& \text { s.t. }\left\{\begin{array}{l}
\mu_{r} \cdot\left(\sum_{q=0}^{2 \tau} Z\left(L_{r r^{\prime}, q}^{D}\right) \cdot p_{r r^{\prime}, q}^{D}-2 \tau\right)+\mu_{r^{\prime}} \cdot \sum_{q=0}^{2 \tau} Z\left(L_{r r^{\prime}, q}^{D}\right) \cdot p_{r r^{\prime}, q}^{D}+\overleftarrow{d}_{r r^{\prime}}^{D}-\vec{d}_{r r^{\prime}}^{D}=0, \quad r, r^{\prime}=1, \ldots, R \\
0<\mu_{r}<1, r=1, \ldots, R-1, \quad \mu_{R}=1 \\
\overleftarrow{d}_{r r^{\prime}}, \vec{d}_{r r^{\prime}}^{D} \geq 0, \overleftarrow{d}_{r r^{\prime}}^{D} \cdot \vec{d}_{r r^{\prime}}^{D}=0, \quad r, r=1, \ldots, R .
\end{array}\right.
\end{aligned}
$$

This proposed model is then solved numerically to produce optimal solutions of the form $\mu^{*}(D)=\left(\mu_{r}^{*} \mid r=1, \ldots, R\right)=\left(\mu^{*}\left(D_{(r)}\right) \mid r=1, \ldots, R\right)$, which are used to calculate discrete Choquet integrals. The comprehensive value $V_{j}$ of selective alternatives $b_{\mathrm{j}}$ is determined from the attribute values $v_{k}\left(y_{j k}\right)$ and the determined fuzzy measures $\mu^{*}(j=1, \ldots, J)$. Larger comprehensive values $V_{j}$ result in better selective alternatives $b_{j}$, which could be ranked using comprehensive values. The optimal selective alternatives can then be identified as exhibiting the largest comprehensive values.

3.5. The Proposed Approach. The proposed target-oriented MADM technique can be summarized as follows:

Step 1. Describe the target-oriented MADM problem. This involves collecting a set of targeted alternatives and selective alternatives. A set of attributes can then be identified and used to make evaluations of decision matrices for the targeted and selective alternatives.

Step 2. Determine the attribute value function for target orientation. The DM is asked to estimate the parameters of attribute value functions based on targeted alternatives. These attribute value functions can be constructed to reflect the DM's integrated preferences for both range and frequency values.

Step 3. Calculate comprehensive values for selective alternatives using discrete Choquet integrals. The corresponding attribute values are then determined using attribute value functions, and the set of fuzzy measures is determined by the DM. Discrete Choquet integrals can be used to calculate comprehensive values for all selective alternatives.

Step 4. Make a target-oriented MADM. In this process, selective alternatives are ranked using the comprehensive values, and the optimal alternative is identified as exhibiting the largest comprehensive value.

\section{Illustrative Example}

Consider a scenario in which three faculty members are applying to be doctoral supervisors in a specific college at a university. The college evaluates their academic performance and ranks them according to the results. This section demonstrates the application of the proposed target-oriented MADM approach in this case.
4.1. Problem Description. Suppose the three applicants $\left\{b_{j} \mid j=1,2,3\right\}$, as part of the evaluation process, are required to refer 16 colleagues $\left\{a_{i} \mid i=1, \ldots, 16\right\}$ who are doctoral supervisors. It is evident that $B=\left\{b_{j} \mid j=1,2,3\right\}$ represents a set of selective alternatives and $A=\left\{a_{i} \mid i=1, \ldots, 16\right\}$ denotes the set of targeted alternatives. The dean of the college, who serves as the decision maker, is invited to submit evaluation results using four different attributes, such as the number of published papers $\left(c_{1}\right)$, the number of longitudinal projects $\left(c_{2}\right)$, the number of graduate supervisors $\left(c_{3}\right)$, and funding for latitudinal projects $\left(c_{4}\right)$. Thus, an attribute set is established as $C=\left\{c_{k} \mid k=1, \ldots, 4\right\}$. The level of attribute $c_{k}$ for targeted alternatives $a_{i}$ and selective alternative $b_{j}$ are collected and denoted as $\left\{x_{i k} \mid i=1, \ldots, 16, k=\right.$ $1, \ldots, 4\}$ and $\left\{y_{j k} \mid j=1,2,3, k=1, \ldots, 4\right\}$. The collected decision information for both targeted and selective alternatives are, respectively, shown in Tables 1 and 2. A decision matrix for $X=\left(x_{i k}\right)_{16 \times 4}$ and $Y=\left(y_{j k}\right)_{3 \times 4}$ can then be established from the information in the table. Note that $x^{k}$ is the reference point for attribute $c_{k}$, set by the DM as a basic condition specified by the university (i.e., $x^{1}=5, x^{2}=1$, $x^{3}=5$, and $\left.x^{4}=40\right)$. These reference points for the four attributes then satisfy $x^{k} \leq x_{1}^{k}, k=1, \ldots, 4$.

4.2. Attribute Value Function Determination. The decision maker is then asked to estimate parameters of the attribute value function for each attribute, based on targeted alternatives.

First, representative data points are selected from the interval $\left[x_{1}^{k}, x_{I_{k}}^{k}\right]$ for attribute $c_{k}$. As seen in Table 1 , the intervals for these four attributes are $\left[x_{1}^{1}, x_{I_{1}}^{1}\right]=[8,25]$, $\left[x_{1}^{2}, x_{I_{2}}^{2}\right]=[1,7], \quad\left[x_{1}^{3}, x_{I_{3}}^{3}\right]=[5,25], \quad$ and $\quad\left[x_{1}^{4}, x_{I_{4}}^{4}\right]=$ $[50,100]$. The number of representative data points in each interval for attributes $c_{1}, c_{2}, c_{3}$, and $c_{4}$ are set as $8,7,13$, and 8 , respectively. These representative data points used to estimate parameters are listed in Table 3.

Second, the representative data are divided into several groups. In this case, 5-category scale denoted by $S=\left\{s_{0}\right.$ : poor, $s_{1}$ : slightly poor, $s_{2}$ : fair, $s_{3}$ : slightly good, and $s_{4}$ : good $\}$ is employed to extract the DM's preference for values of attribute level. The number of groups for each attribute is determined using $G_{k}=\operatorname{roundup}\left(\left(N_{k}-1\right) / 2 \tau\right)$. Specifically, $G_{1}=\operatorname{roundup}((8-1) / 4)=2, G_{2}=\operatorname{roundup}((7-1) / 4)=2$, $G_{3}=\operatorname{roundup}((13-1 / 4))=3$, and $G_{4}=$ roundup $((8-1) / 4 / 4)=2$. The set of data points in the $g_{k}$ group $X_{g_{k}}^{k}=\left(x_{t}^{g_{k}} \mid t=1, \ldots, T_{g_{k}}\right)$ is determined from 
TABLE 1: Decision information for targeted alternatives.

\begin{tabular}{|c|c|c|c|c|}
\hline Alternative & $c_{1}$ & $c_{2}$ & $c_{3}$ & $c_{4}$ \\
\hline$a_{1}$ & 13 & 3 & 8 & 70 \\
\hline$a_{2}$ & 13 & 2 & 12 & 80 \\
\hline$a_{3}$ & 8 & 2 & 5 & 60 \\
\hline$a_{4}$ & 12 & 4 & 12 & 100 \\
\hline$a_{5}$ & 9 & 3 & 14 & 60 \\
\hline$a_{6}$ & 20 & 3 & 20 & 80 \\
\hline$a_{7}$ & 15 & 2 & 15 & 60 \\
\hline$a_{8}$ & 13 & 2 & 10 & 70 \\
\hline$a_{9}$ & 10 & 2 & 20 & 65 \\
\hline$a_{10}$ & 17 & 1 & 25 & 50 \\
\hline$a_{11}$ & 25 & 2 & 18 & 90 \\
\hline$a_{12}$ & 17 & 4 & 22 & 50 \\
\hline$a_{13}$ & 18 & 7 & 8 & 80 \\
\hline$a_{14}$ & 20 & 5 & 20 & 100 \\
\hline$a_{15}$ & 10 & 1 & 6 & 60 \\
\hline$a_{16}$ & 13 & 5 & 8 & 80 \\
\hline
\end{tabular}

TABLe 2: Decision information for selective alternatives.

\begin{tabular}{lcccc}
\hline Alternative & $c_{1}$ & $c_{2}$ & $c_{3}$ & \\
\hline$b_{1}$ & 8 & 2 & 15 & 60 \\
$b_{2}$ & 10 & 4 & 14 & 85 \\
$b_{3}$ & 12 & 3 & 18 & 80 \\
\hline
\end{tabular}

$g_{k}=1, \ldots, G_{k}(k=1,2,3$, and 4). For instance, all of the attributes in $\left\{x_{i 1} \mid i=1, \ldots, 8\right\}=\{8,10, \ldots, 25\}$ are selected as representative data points for attribute $c_{1}$. This set of data is then divided into two groups: $X_{1}^{1}=\left(x_{1}^{1}, \ldots, x_{5}^{1}\right)=$ $(8,10,12,14,16)$ and $X_{2}^{1}=\left(x_{5}^{1}, \ldots, x_{8}^{1}\right)=(16,19,22,25)$.

Third, the judgment matrix for each group of points is constructed with PLPR. The probability distribution $\pi_{k}=$ $\left[p\left(x_{1}^{k}\right), \ldots, p\left(x_{I_{k}}^{k}\right)\right]$ is easily calculated from the data in
Table 1 and is shown in Figure 2. Taking this probability distribution $\pi_{k}$ as a decision criterion, the DM is then invited to make pairwise comparisons of the values of data points existing in the group $X_{g_{k}}^{k}=\left(x_{t}^{g_{k}} \mid t=1, \ldots, T_{g_{k}}\right)$. The DM's judgment matrix can then be constructed using equation (13). For instance, the judgment matrix $L_{g_{2}}=\left(L_{t t^{\prime}}^{g_{k}}(p)\right)_{T_{g_{2}} \times T_{g_{2}}}$ of group $X_{g_{2}}^{2}$ for attribute $c_{2}$ is given by

$$
L_{g_{2}}=\left(L_{t t^{\prime}}^{g_{2}}(p)\right)_{3 \times 3}=\left(\begin{array}{ccc}
\left\{s_{2}(1)\right\} & \left\{s_{2}(1)\right\} & \left\{s_{1}(0.1), s_{2}(0.9)\right\} \\
\left\{s_{2}(1)\right\} & \left\{s_{2}(1)\right\} & \left\{s_{1}(0.1), s_{2}(0.9)\right\} \\
\left\{s_{3}(0.1), s_{2}(0.9)\right\} & \left\{s_{3}(0.1), s_{2}(0.9)\right\} & \left\{s_{2}(1)\right\}
\end{array}\right) .
$$

Fourth, a parameter estimation model for attribute value functions is constructed by substituting the given judgment matrix into equations (18a) and (18b). The Lingo 11.0 software package is used to solve the parameter estimation model and determine the four attribute parameters (i.e., $\xi_{1}^{*}=0.2, \alpha_{1}^{*}=0.31, \vartheta_{1}^{*}=0.54 ; \xi_{2}^{*}=0.5, \alpha_{2}^{*}=0.22, \vartheta_{2}^{*}=0.27$;

$\xi_{3}^{*}=0.07, \alpha_{3}^{*}=0.9, \quad \vartheta_{3}^{*}=0.07 ; \xi_{4}^{*}=0.01, \alpha_{4}^{*}=0.99$, and $\left.\vartheta_{4}^{*}=0.49\right)$. The resulting attribute value functions are given by

$$
\begin{cases}v_{1}\left(x_{i 1}\right)=0.2\left(x_{i 1}-5\right)^{0.31}+0.54 F_{1}\left(x_{i 1} \mid \pi_{1}\right), & x_{i 1} \in[8,25], \\ v_{2}\left(x_{i 2}\right)=0.5\left(x_{i 2}-1\right)^{0.22}+0.27 F_{2}\left(x_{i 2} \mid \pi_{2}\right), & x_{i 2} \in[1,7], \\ v_{3}\left(x_{i 3}\right)=0.07\left(x_{i 3}-5\right)^{0.9}+0.07 F_{3}\left(x_{i 3} \mid \pi_{3}\right), & x_{i 3} \in[5,25], \\ v_{4}\left(x_{i 4}\right)=0.01\left(x_{i 4}-40\right)^{0.99}+0.49 F_{4}\left(x_{i 4} \mid \pi_{4}\right), & x_{i 4} \in[50,100] .\end{cases}
$$


TABLE 3: Representative data points for various attributes.

\begin{tabular}{lccccccccccc}
\hline Attribute & 1 & 2 & 3 & 4 & 5 & 6 & 7 & 8 & 9 & 10 & 11 \\
\hline$c_{1}$ & 8 & 10 & 12 & 14 & 16 & 19 & 22 & 25 & & & \\
$c_{2}$ & 1 & 2 & 3 & 4 & 5 & 6 & 7 & & & & \\
$c_{3}$ & 5 & 6 & 7 & 8 & 10 & 12 & 14 & 16 & 18 & 20 & 22 \\
$c_{4}$ & 50 & 60 & 65 & 70 & 75 & 80 & 90 & 100 & & 23 \\
\hline
\end{tabular}

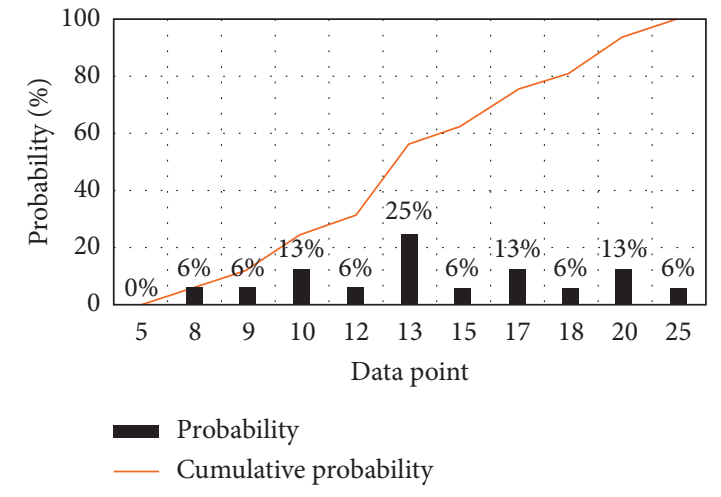

(a)

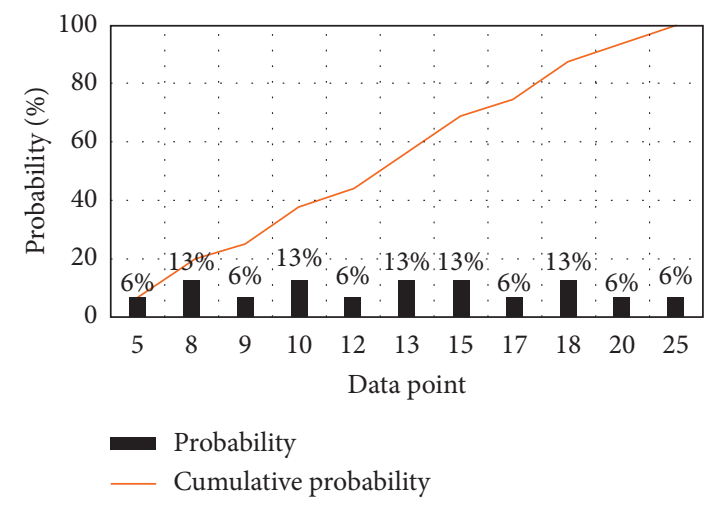

(c)

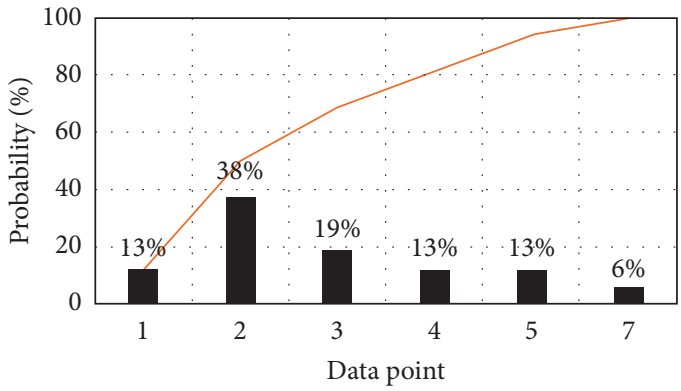

Probability

_ Cumulative probability

(b)

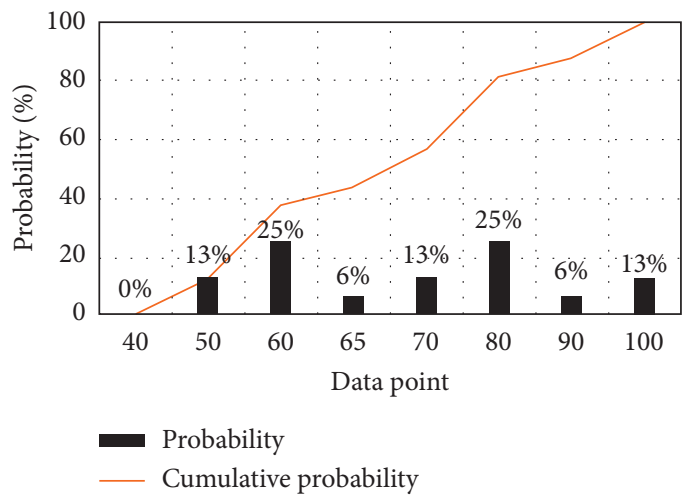

(d)

Figure 2: Probability distributions on attributes. (a) Attribute $c_{1}$. (b) Attribute $c_{2}$. (c) Attribute $c_{3}$. (d) Attribute $c_{4}$.

4.3. Comprehensive Value Calculation and Decision-Making. The values of selective alternatives $B=\left\{b_{j} \mid j=1, \ldots, 3\right\}$ for attribute $c_{k}$ are calculated using $v_{k}\left(y_{j k}\right)=\xi_{k}^{*} \cdot\left(y_{j k}-\right.$ $\left.x^{k}\right)^{\alpha_{k}^{*}}+\vartheta_{k}^{*} \cdot F_{k}\left(y_{j k} \mid \pi_{k}\right)$, where $F_{k}\left(y_{j k} \mid \pi_{k}\right)$ and the determined parameters are shown in equations (19) and (24), respectively. For instance, the attribute value for the selective alternative $b_{1}$ of attribute $c_{2}$ is calculated using $v_{2}\left(y_{12}\right)=$ $0.5 \times(2-1)^{0.22}+0.27 \times 0.5=0.63$. The attribute values for selective alternatives are shown in Table 4.

The use of discrete Choquet integrals to determine attribute values requires the estimation of fuzzy measure sets.
In this case, the set of fuzzy measures to be calculated is given by

$$
\begin{aligned}
\mu(D)= & \left(\mu_{1}\left(c_{2}\right), \mu_{2}\left(c_{2}, c_{3}\right), \mu_{3}\left(c_{2}, c_{4}\right), \mu_{4}\left(c_{2}, c_{3}, c_{4}\right),\right. \\
& \left.\mu_{5}\left(c_{1}, c_{2}, c_{3}, c_{4}\right)\right) .
\end{aligned}
$$

A 5-category scale $S=\left\{s_{0}\right.$ : poor, $s_{1}$ : slightly poor, $s_{2}$ : fair, $s_{3}$ : slightly good, $s_{4}$ : good $\}$ is also used to express varying degrees of preference. The DM is then invited to provide a preference degree for the fuzzy measures $\mu_{r}$ over $\mu_{r^{\prime}}$. The use 
TABLe 4: Attribute values for selective alternatives.

\begin{tabular}{lcccc}
\hline Alternative & $c_{1}$ & $c_{2}$ & $c_{3}$ & $c_{4}$ \\
\hline$b_{1}$ & 0.32 & 0.63 & 0.57 & 0.35 \\
$b_{2}$ & 0.47 & 0.85 & 0.52 & 0.78 \\
$b_{3}$ & 0.54 & 0.76 & 0.72 & 0.74 \\
\hline
\end{tabular}

of PLPR yields $L_{r r^{\prime}}^{D}(p)\left(r, r^{\prime}=1, \ldots, 5\right)$. The judgment matrix for estimating fuzzy measures can then be expressed as

$$
L_{D}=\left(L_{r r^{\prime}}^{D}(p)\right)_{5 \times 5}=\left(\begin{array}{cccccc}
\left\{s_{2}(1)\right\} & \left\{s_{1}(0.6), s_{2}(0.4)\right\} & \left\{s_{1}(0.5), s_{2}(0.5)\right\} & \left\{s_{0}(0.1), s_{1}(0.9)\right\} & \left\{s_{0}(0.4), s_{1}(0.6)\right\} \\
\left\{s_{3}(0.6), s_{2}(0.4)\right\} & \left\{s_{2}(1)\right\} & \left\{s_{2}(0.9), s_{3}(0.1)\right\} & \left\{s_{1}(0.3), s_{2}(0.7)\right\} & \left\{s_{1}(1)\right\} \\
\left\{s_{3}(0.5), s_{2}(0.5)\right\} & \left\{s_{2}(0.9), s_{1}(0.1)\right\} & \left\{s_{2}(1)\right\} & \left\{s_{1}(0.6), s_{2}(0.4)\right\} & \left\{s_{0}(0.1), s_{1}(0.9)\right\} \\
\left\{s_{4}(0.1), s_{3}(0.9)\right\} & \left\{s_{3}(0.3), s_{2}(0.7)\right\} & \left\{s_{3}(0.6), s_{2}(0.4)\right\} & \left\{s_{2}(1)\right\} & \left\{s_{1}(0.5), s_{2}(0.5)\right\} \\
\left\{s_{4}(0.4), s_{3}(0.6)\right\} & \left\{s_{3}(1)\right\} & \left\{s_{4}(0.1), s_{3}(0.9)\right\} & \left\{s_{3}(0.5), s_{2}(0.5)\right\} & \left\{s_{2}(1)\right\}
\end{array}\right) .
$$

A fuzzy measure determination model is developed by substituting the judgment matrix $L_{D}$ into equation (22). The Lingo 11.0 software package is used to solve the constructed model for the fuzzy measure attributes, expressed as

$$
\mu(D)=\left\{\begin{array}{l}
\mu_{1}\left(c_{2}\right)=0.18 \\
\mu_{2}\left(c_{2}, c_{3}\right)=0.33 \\
\mu_{3}\left(c_{3}, c_{4}\right)=0.3 \\
\mu_{4}\left(c_{2}, c_{3}, c_{4}\right)=0.6 \\
\mu_{5}\left(c_{1}, c_{2}, c_{3}, c_{4}\right)=1
\end{array}\right.
$$

Comprehensive values for three selective alternatives are determined from the attribute values $v_{k}\left(y_{j k}\right)$ listed in Table 4. This is done for all attributes by substituting the determined fuzzy measures $\mu^{*}$ into equation (20). For instance, the comprehensive value $V_{1}$ for the selective alternative $b_{1}$ is determined by $V_{1}=0.63 \times 0.18+0.57 \times($ $0.33-0.18)+0.35 \times(0.6-0.33)+0.32 \times(1-0.6)=0.42$. The resulting comprehensive values for the selective alternatives $b_{2}$ and $b_{3}$ are $V_{2}=0.59$ and $V_{3}=0.66$, respectively. The ranking of selective alternatives (applicants) is then $b_{3}>b_{2}>b_{1}$, with $b_{3}$ as the optimal choice.

\section{Conclusion}

MADM is widely applied in a variety of fields. It can be used to make choices and identify the best option from among a finite set of decision alternatives, using multiple attributes. The problem of uncertainty in subjective preferences can be solved using fuzzy sets and corresponding extensions. Unfortunately, existing methods establish to evaluate alternatives by comparing them with each other, but cannot solve target-oriented decision problems that include both selective and targeted alternatives. In this study, a target- oriented MADM approach is proposed based on PLPR. An illustrative example is introduced to demonstrate the viability of the proposed techniques.

This study contributes primary in the following three aspects: first, attribute values for selective alternatives are determined by referring to targeted alternatives. MADM can then be conducted to achieve target orientation. Second, the attribute value function for alternatives of each attribute is constructed based on RFT and the CPT. The result can represent not only the range value but also the frequency value. Third, a parameter estimation model and fuzzy measure estimation model are both established using PLPR to determine the relevant parameters involved in a targetoriented MADM process. An illustrative example is included to demonstrate the superiority of the proposed approach. Since PLPR can directly extract the DM's preference information, range and frequency values can both be included in the attribute value function. This serves as a basis for target orientation in MADM. In addition, the parameters involved in the attribute value function and the discrete Choquet integral can be reasonably estimated using established mathematical programming models. Consequently, it is believed the proposed approach can accurately solve target-oriented MADM problems.

This study investigates target-oriented MADM under the assumption that attribute levels of selective alternatives are between the minimum and maximum levels of targeted alternatives. Relaxing this assumption will be pursued in future studies.

\section{Data Availability}

The data used to support the findings of this study are included within the article. 


\section{Conflicts of Interest}

The authors declare that they have no conflicts of interest.

\section{Acknowledgments}

This research was supported by the Humanities and Social Science Foundation of Ministry of Education of China under Grant no. 20YJA630028.

\section{References}

[1] M. Grabisch, C. Labreuche, and M. Ridaoui, "On importance indices in multicriteria decision making," European Journal of Operational Research, vol. 277, no. 1, pp. 269-283, 2019.

[2] F. Haag, J. Lienert, N. Schuwirth, and P. Reichert, "Identifying non-additive multi-attribute value functions based on uncertain indifference statements," Omega, vol. 85, pp. 49-67, 2019.

[3] M. Lin, C. Huang, and Z. Xu, "Topsis method based on correlation coefficient and entropy measure for linguistic pythagorean fuzzy sets and its application to multiple attribute decision making," Complexity, vol. 2019, Article ID 6967390, 16 pages, 2019.

[4] G. F. Can and S. Demirok, "Universal usability evaluation by using an integrated fuzzy multi criteria decision making approach," International Journal of Intelligent Computing and Cybernetics, vol. 12, no. 2, pp. 194-223, 2019.

[5] W. Liang, M. Goh, and Y.-M. Wang, "Multi-attribute group decision making method based on prospect theory under hesitant probabilistic fuzzy environment," Computers \& Industrial Engineering, vol. 149, p. 106804, 2020.

[6] H. Li, L. Lv, F. Li, L. Wang, and Q. Xia, "A novel approach to emergency risk assessment using FMEA with extended MULTIMOORA method under interval-valued pythagorean fuzzy environment," International Journal of Intelligent Computing and Cybernetics, vol. 13, no. 1, pp. 41-65, 2020.

[7] M. Qiyas, M. A. Khan, S. Khan, and S. Abdullah, "Concept of yager operators with the picture fuzzy set environment and its application to emergency program selection," International Journal of Intelligent Computing and Cybernetics, vol. 13, no. 4, pp. 455-483, 2020.

[8] M. Lin, J. Wei, Z. Xu, and R. Chen, "Multiattribute group decision-making based on linguistic pythagorean fuzzy interaction partitioned bonferroni mean aggregation operators," Complexity, vol. 2018, Article ID 9531064, 24 pages, 2018.

[9] M. Lin, H. Wang, Z. Xu, Z. Yao, and J. Huang, "Clustering algorithms based on correlation coefficients for probabilistic linguistic term sets," International Journal of Intelligent Systems, vol. 33, no. 12, pp. 2402-2424, 2018.

[10] L. A. Zadeh, "The concept of a linguistic variable and its application to approximate reasoning-I," Information Science, vol. 3, no. 8, pp. 199-249, 1975.

[11] V. Torra, "Hesitant fuzzy sets," International Journal of Intelligent Systems, vol. 6, no. 25, pp. 529-539, 2010.

[12] R. M. Rodríguez, L. Martínez, and F. Herrera, "A group decision making model dealing with comparative linguistic expressions based on hesitant fuzzy linguistic term sets," Information Sciences, vol. 241, pp. 28-42, 2013.

[13] P. Li, J. Liu, Y. Yang, and C. Wei, "Evaluation of povertystricken families in rural areas using a novel case-based reasoning method for probabilistic linguistic term sets," Computers \& Industrial Engineering, vol. 147, p. 106658, 2020.
[14] P. Li and C. Wei, "An emergency decision-making method based on D-S evidence theory for probabilistic linguistic term sets," International Journal of Disaster Risk Reduction, vol. 37, p. 101178, 2019.

[15] Q. Pang, H. Wang, and Z. Xu, "Probabilistic linguistic term sets in multi-attribute group decision making," Information Sciences, vol. 369, pp. 128-143, 2016.

[16] M. Lin, C. Huang, Z. Xu, and R. Chen, "Evaluating IoT platforms using integrated probabilistic linguistic MCDM method," Ieee Internet of Things Journal, vol. 7, no. 11, pp. 11195-11208, 2020.

[17] L. Jiang and H. Liao, "Network consensus analysis of probabilistic linguistic preference relations for group decision making and its application in urban household waste classification," Journal of Cleaner Production, vol. 278, Article ID 122766, 2021.

[18] F. Herrera, E. Herrera-Viedma, and J. L. Verdegay, "A model of consensus in group decision making under linguistic assessments," Fuzzy Sets and Systems, vol. 78, no. 1, pp. 73-87, 1996.

[19] X. Xu, L. Wang, X. Chen, and B. Liu, "Large group emergency decision-making method with linguistic risk appetites based on criteria mining," Knowledge-Based Systems, vol. 182, Article ID 104849, 2019.

[20] T.-H. Chang, S.-C. Hsu, T.-C. Wang, and C.-Y. Wu, "Measuring the success possibility of implementing ERP by utilizing the incomplete linguistic preference relations," Applied Soft Computing, vol. 12, no. 5, pp. 1582-1591, 2012.

[21] C. Tan, D. D. Wu, and B. Ma, "Group decision making with linguistic preference relations with application to supplier selection," Expert Systems with Applications, vol. 38, no. 12, pp. 14382-14389, 2011.

[22] B. Zhu and Z.-S. Xu, "Consistency measures for hesitant fuzzy linguistic preference relations," Transactions on Fuzzy Systems, vol. 1, no. 22, pp. 35-45, 2014.

[23] C.-C. Li, R. M. Rodríguez, L. Martínez, Y. Dong, and F. Herrera, "Consistency of hesitant fuzzy linguistic preference relations: an interval consistency index," Information Sciences, vol. 432, pp. 347-361, 2018.

[24] H. Liu, Y. Ma, and L. Jiang, "Managing incomplete preferences and consistency improvement in hesitant fuzzy linguistic preference relations with applications in group decision making," Information Fusion, vol. 51, pp. 19-29, 2019.

[25] Z. Zhang and C. Wu, "On the use of multiplicative consistency in hesitant fuzzy linguistic preference relations," KnowledgeBased Systems, vol. 72, pp. 13-27, 2014.

[26] Y. Zhang, Z. Xu, H. Wang, and H. Liao, "Consistency-based risk assessment with probabilistic linguistic preference relation," Applied Soft Computing, vol. 49, pp. 817-833, 2016.

[27] R.-X. Nie and J.-Q. Wang, "Prospect theory-based consistency recovery strategies with multiplicative probabilistic linguistic preference relations in managing group decision making," Arabian Journal for Science and Engineering, vol. 45, no. 3, pp. 2113-2130, 2020.

[28] Y. Zhang, Z. Xu, and H. Liao, “A consensus process for group decision making with probabilistic linguistic preference relations," Information Sciences, vol. 414, pp. 260-275, 2017.

[29] Y. Song and J. Hu, "Large-scale group decision making with multiple stakeholders based on probabilistic linguistic preference relation," Applied Soft Computing, vol. 80, pp. 712-722, 2019.

[30] J. Gao, Z. Xu, P. Ren, and H. Liao, “An emergency decision making method based on the multiplicative consistency of 
probabilistic linguistic preference relations," International Journal of Machine Learning and Cybernetics, vol. 10, no. 7, pp. 1613-1629, 2019.

[31] Y.-W. Du, Y.-M. Wang, and M. Qin, "New evidential reasoning rule with both weight and reliability for evidence combination," Computers \& Industrial Engineering, vol. 124, pp. 493-508, 2018.

[32] M. Lin, Z. Xu, Y. Zhai, and Z. Yao, "Multi-attribute group decision-making under probabilistic uncertain linguistic environment," Journal of the Operational Research Society, vol. 69, no. 2, pp. 157-170, 2017.

[33] R. F. Bordley and C. W. Kirkwood, "Multiattribute preference analysis with performance targets," Operations Research, vol. 52, no. 6, pp. 823-835, 2004.

[34] N. Stewart, S. Reimers, and A. J. L. Harris, "On the origin of utility, weighting, and discounting functions: how they get their shapes and how to change their shapes," Management Science, vol. 61, no. 3, pp. 687-705, 2015.

[35] R. W. Niedrich, S. Sharma, and D. H. Wedell, "Reference price and price perceptions: a comparison of alternative models," Journal of Consumer Research, vol. 28, no. 3, pp. 339-354, 2001.

[36] R. W. Niedrich, D. Weathers, R. C. Hill, and D. R. Bell, "Specifying price judgments with range-frequency theory in models of brand choice," Journal of Marketing Research, vol. 46, no. 5, pp. 693-702, 2009.

[37] Z. S. Xu, "EOWA and EOWG operators for aggregating linguistic labels based on linguistic preference relations," International Journal of Uncertainty, Fuzziness and Knowledge-Based Systems, vol. 12, no. 6, pp. 791-810, 2004.

[38] M. Delgado, J. L. Verdegay, and M. A. Vila, "On aggregation operations of linguistic labels," International Journal of Intelligent Systems, vol. 8, no. 3, pp. 351-370, 1993.

[39] Z. Xu, "Incomplete linguistic preference relations and their fusion," Information Fusion, vol. 7, no. 3, pp. 331-337, 2006.

[40] M. Lin, Z. Chen, H. Liao, and Z. Xu, "ELECTRE II method to deal with probabilistic linguistic term sets and its application to edge computing," Nonlinear Dynamics, vol. 96, no. 3, pp. 2125-2143, 2019.

[41] A. Parducci, Happiness, Pleasure, and Judgment: The Contextual Theory and Its Applications, L. Erlbaum Associates, Mahwah, NJ, USA, 1995.

[42] A. Tversky and D. Kahneman, "Advances in prospect theory: cumulative representation of uncertainty," Journal of Risk and Uncertainty, vol. 5, no. 4, pp. 297-323, 1992.

[43] A. S. Booij, B. M. S. van Praag, and G. van de Kuilen, "A parametric analysis of prospect theory's functionals for the general population," Theory and Decision, vol. 68, no. 1-2, pp. 115-148, 2010.

[44] T. Pachur, R. S. Suter, and R. Hertwig, "How the twain can meet: prospect theory and models of heuristics in risky choice," Cognitive Psychology, vol. 93, pp. 44-73, 2017.

[45] M. Grabisch, I. Kojadinovic, and P. Meyer, "A review of methods for capacity identification in Choquet integral based multi-attribute utility theory," European Journal of Operational Research, vol. 186, no. 2, pp. 766-785, 2008.

[46] Y. Ju, X. Liu, and A. Wang, "Some new shapley 2-tuple linguistic choquet aggregation operators and their applications to multiple attribute group decision making," Soft Computing, vol. 20, no. 10, pp. 4037-4053, 2016.

[47] M. Grabisch, "The application of fuzzy integrals in multicriteria decision making," European Journal of Operational Research, vol. 89, no. 3, pp. 445-456, 1996.
[48] G. N. Büyüközkan and D. Ruan, "Choquet integral based aggregation approach to software development risk assessment," Information Sciences, vol. 180, no. 3, pp. 441-451, 2010.

[49] Y.-W. Du and X.-X. Li, "Hierarchical dematel method for complex systems," Expert Systems with Applications, Article ID 113871, 2020.

[50] Y.-W. Du and J.-J. Zhong, "Generalized combination rule for evidential reasoning approach and Dempster-Shafer theory of evidence," Information Sciences, vol. 547, pp. 1201-1232, 2021.

[51] Y.-W. Du and J.-J. Zhong, "Group inference method of attribution theory based on Dempster-Shafer theory of evidence," Knowledge-Based Systems, vol. 188, Article ID 104985, 2020.

[52] K. Chen, P. Chen, L. Yang, and L. Jin, "Grey clustering evaluation based on AHP and interval grey number," International Journal of Intelligent Computing and Cybernetics, vol. 12, no. 1, pp. 127-137, 2019.

[53] C. Labreuche, "An axiomatization of the choquet integral in the context of multiple criteria decision making without any commensurability assumption," Annals of Operations Research, vol. 271, no. 2, pp. 701-735, 2018.

[54] D. Joshi and S. Kumar, "Interval-valued intuitionistic hesitant fuzzy choquet integral based topsis method for multi-criteria group decision making," European Journal of Operational Research, vol. 248, no. 1, pp. 183-191, 2016.

[55] J.-L. Marichal and M. Roubens, "Determination of weights of interacting criteria from a reference set," European Journal of Operational Research, vol. 124, no. 3, pp. 641-650, 2000. 